\title{
Unexpected Detection of Parotid Gland Malignancy during Primary Extracapsular Dissection
}

\author{
Konstantinos Mantsopoulos, PhD, MD', Stylianos Velegrakis, MD', \\ and Heinrich Iro, PhD, MD'
}

No sponsorships or competing interests have been disclosed for this article.

\begin{abstract}
Objective. To evaluate the oncologic and functional outcome in cases in which a false preoperative working hypothesis "benign parotid tumor" led to a primary extracapsular dissection being performed rather than a traditional, more radical surgical modality.
\end{abstract}

Study Design. Case series with chart review.

Setting. Academic tertiary referral center.

Subjects and Methods. The records of all patients treated for malignant tumors of the parotid gland between 2006 and 2012 were retrospectively studied. Patients were excluded from our study sample for insufficient data or if their histories indicated manifestation of malignant tumors without primary parotid origin (squamous cell carcinomas, lymphomas, melanomas), revision surgery, as well as concomitant or past malignant tumors. Consequently, a total of 29 patients with 30 primary malignant tumors of the parotid gland formed our study sample (I 4 men, I 5 women; male:female ratio, 0.93:I; mean age, 55.4 years; range, 14-85 years).

Results. Of 30 cases, 22 had low-grade tumors. Further tumor cells were detected in only the histology specimens of the parotid in 3 of the 25 cases treated subsequently with completion parotidectomy. Five patients received adjuvant radiation. Five-year disease-specific survival was $100 \%$; local disease control was $96.6 \%$. Of 30 cases, 28 had HouseBrackmann I after tumor treatment; the other 2 cases had a slight paralysis (House-Brackmann II).

Conclusion. Beginning with the "false" working hypothesis and performing an extracapsular dissection in unsuspected cases seems to have no adverse impact on patients' survival and postoperative quality of life in cases in which definitive histology reveals malignancy.

\section{Keywords}

benign tumors, parotid gland, extracapsular dissection, survival, facial nerve
Received October 10, 20I4; revised February 13, 20I5; accepted February 27, 2015.

$\mathrm{P}$ rimary malignancies of the parotid gland are relatively rare, having an annual worldwide incidence rate of $<2$ per $100,000 .{ }^{1}$ Treatment of this disease remains challenging because of its extremely low incidence, the 24 histologic types, ${ }^{2}$ and accordingly varied biological behavior. ${ }^{3}$ There is a continued debate as to the extent of parotidectomy ${ }^{4,5}$ and the indication for elective neck dissection by $\mathrm{cN} 0$ neck status ${ }^{6-8}$ in cases with a histologically confirmed primary malignant tumor of the parotid gland.

It is, however, a common experience that the majority of these patients present with clinically and radiologically unsuspected lesions. Lack of suspected malignancy leads, in many cases, to a more conservative form of surgical treatment ${ }^{9}$ : in cases in which specific clinical criteria (unilocularity, location, depth, mobility at surgery after raising the skin flap) and radiologic criteria (well-defined tumor margins, lack of enlarged cervical lymph nodes) are fulfilled, an extracapsular dissection has increasingly been performed in our department over the last 12 years. Over these years, 1 question has progressively emerged as a part of internal audit: Is it actually that bad for the patient if a malignant parotid gland tumor is being primarily managed by an extracapsular dissection? If the potential risk in this surgical modality of encountering a malignant tumor masquerading as a benign lesion is high and the subsequent course of the disease is adversely affected, this would prohibit the use of extracapsular dissection as a viable alternative to more extensive modalities for a simple parotid lump. ${ }^{9}$

The main question of our study was to what extent the primary extracapsular dissection of a primarily unsuspected lesion in the parotid gland might be associated with compromises in

'Department of Otorhinolaryngology, Head and Neck Surgery, FriedrichAlexander-Universität Erlangen-Nürnberg (FAU), Erlangen, Germany

Corresponding Author:

Konstantinos Mantsopoulos, PhD, MD, Department of Otorhinolaryngology, Head and Neck Surgery, Friedrich-Alexander-Universität Erlangen-

Nürnberg (FAU), Waldstrasse I, Erlangen, 91054, Germany.

Email: konstantinos.mantsopoulos@uk-erlangen.de 
the oncologic and functional outcome in cases in which definitive pathology unexpectedly reveals a malignancy. If no compromises could be detected, this might be an argument for the value of extracapsular dissection as a primary diagnostic tool as well as perhaps a primary surgical therapy for certain lowgrade, low-stage parotid gland malignancies.

\section{Methods}

This retrospective study was conducted at an academic tertiary referral center specializing in salivary gland pathologies (Department of Otorhinolaryngology, Head and Neck Surgery, Friedrich-Alexander University Erlangen-Nürnberg (FAU), Erlangen, Germany). The records of all patients treated for malignant tumors of the parotid gland between 2006 and 2012 were retrospectively studied. Patients were excluded from our study sample for insufficient data or if their histories indicated manifestation of malignant tumors without primary salivary gland origin (squamous cell carcinomas, lymphomas, melanomas) in the parotid gland, revision surgery, and other malignant tumors. Approval was obtained from the institutional review board of our hospital (Clinical Ethics Committee, University Hospital Erlangen).

All patients were evaluated preoperatively as wells as postoperatively with a clinical and ultrasound examination of the head and neck region and, in some cases, computed tomography or magnetic resonance imaging. Preoperatively, the function of the facial nerve was clinically assessed using the House-Brackmann grading system ${ }^{10}$ and by electromyography of the facial nerve in all cases.

Our primary survey detected 165 patients treated for malignant tumors of the parotid gland. The majority of cases in this initial group were patients either with preoperatively known malignancy (and complete parotidectomy with neck dissection in all cases) or with confirmation of malignancy only on intraoperative frozen sections and then completion parotidectomy with neck dissection - after intraoperative discussion with the pathologist - in a single procedure. Remarkably, the rest $(n=29)$ consisted of patients who underwent primary extracapsular dissection of the tumor because of a lack of pre- or intraoperative suspicion of malignancy. These were cases with a single and mobile lesion with preoperative clinical and ultrasound signs of a benign tumor and a superficial location within the lateral lobe of the parotid gland or, rarely, lesions arising from the pharyngeal prolongation of the parotid gland and location in the deep parapharyngeal space (extracapsular dissection via extended submandibular approach). Because of good dissection circumstances, lack of infiltrative behavior, and lack of suspicion of malignancy during surgery, frozen section was not performed in such cases. An extracapsular dissection was defined as removal of a tumor, respect to its capsule without exposure of the main trunk of the facial nerve. ${ }^{11}$

The aim of our study was to evaluate the oncologic and functional (permanent facial palsy) outcome in cases in which a false preoperative working hypothesis "benign parotid tumor" led to a primary extracapsular dissection being performed rather than a traditional, more radical surgical modality. The oncologic

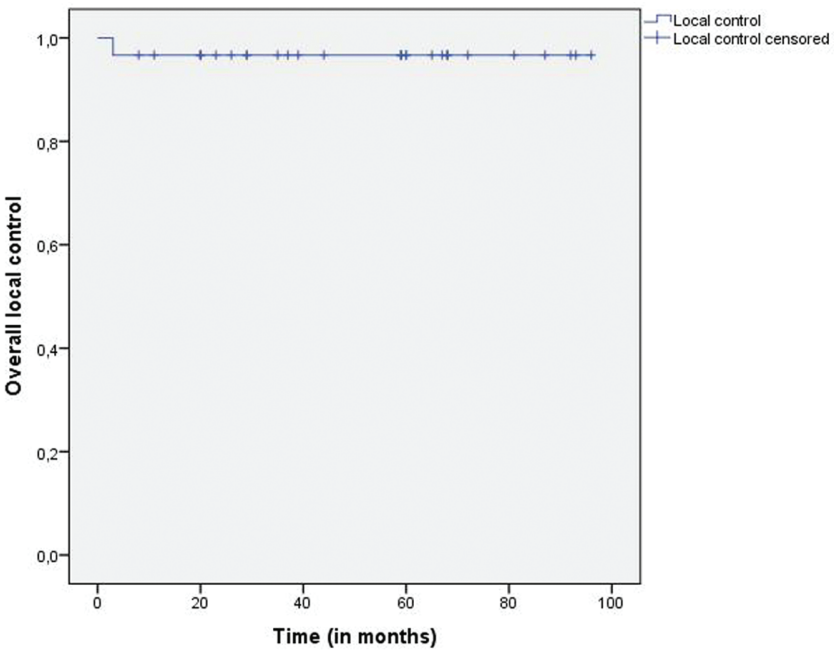

Figure I. Kaplan-Meier analysis of the overall local control of the study cases.

outcome was measured in terms of 5-year disease-specific survival rate and local disease control rate. Five-year diseasespecific survival rate was defined as the percentage of patients who did not die of the tumor of interest within 5 years, divided by the total number of patients. Local disease control reflects tumor recurrence at the primary site and was calculated from the date of surgery to the date when local recurrence was diagnosed or to the date of the last follow-up. Statistical analysis was performed using the Kaplan-Meier method with 95\% confidence intervals.

\section{Results}

Our primary survey detected 165 patients treated for malignant tumors of the parotid gland during the period of the study. From this initial group, 56 patients received a primary extracapsular dissection for unsuspected parotid malignancy. Twenty-two of 56 patients had a malignant tumor without salivary gland origin (squamous cell carcinoma, lymphoma, melanoma) and were excluded from our study. Four other patients were excluded because of extracapsular revision surgery and other concomitant or past malignant tumors in their patient histories. Consequently, a total of 29 patients with 30 primary malignant tumors of the parotid gland formed our study sample (14 men, 15 women; male:female ratio, 0.93:1). Their mean age was 55.4 years (14-85 years). Information on clinical and ultrasound findings, tumor pathology (histologic type and TNM classification), further therapy after primary extracapsular dissection, as well as oncologic and functional outcome after completion of tumor treatment are shown in Supplemental Table S1 (see appendix at www.otojournal.org). Five-year disease specific survival was $100 \%$; local disease control was 96.6\% (95\% confidence interval: 92.9\%-98.9\%; Figure I).

\section{Discussion}

Our primary purpose is to perform a safe and oncologically adequate procedure with the smallest possible discomfort 
for the patient. In our department, all patients are evaluated preoperatively with a clinical and ultrasound examination of the head and neck region and, in some cases, computed tomography or magnetic resonance imaging. Pain in the parotid region, facial palsy, or fixation to skin may point toward malignancy. In sonography, blurred, poorly defined margins and a heterogeneous echotexture may indicate malignancy. In ultrasound elastography, "garland signs" are more frequent in malignant than in benign parotid tumors. In the event of preoperative suspicion of parotid gland malignancy, fine-needle aspiration cytology (FNAC) or core needle biopsy (CNB) of the suspect lesion is performed in our department. In the case of histopathologic confirmation of malignancy, an interdisciplinary tumor board takes place to define the best possible treatment. In cases of preoperatively known primary malignant tumors of the parotid gland, a complete parotidectomy with neck dissection is performed. If there is clinical or electromyographic evidence of tumor infiltration of the facial nerve or its branches, a radical parotidectomy is performed. The same treatment modality is chosen in cases in which primary parotid gland malignancy is detected solely on frozen sections. In agreement with the literature, ${ }^{12}$ this surgical concept allows complete removal of the primary tumor and all possible locoregional metastatic foci to intraparotid and cervical lymph nodes. Stennert et al found a high incidence of occult metastatic disease in the lymph nodes of the deep lobe and recommended a complete parotidectomy in every case of primary parotid gland carcinomas. ${ }^{8}$ In a study with 128 mucoepidermoid carcinomas, most recurrences were detected in the parotid bed, pointing to the need for adequate control, at least at the site of the primary tumor. ${ }^{12}$

In our experience, however, a malignant tumor of the parotid gland does not necessarily have any clinical (pain, facial palsy) or imaging signs of malignancy (enlarged neck nodes, blurred lesion margins, elastographic "garland" $\operatorname{sign}^{13}$ ). According to Digonnet et al, the most common histologic types (acinic cell carcinoma, low-grade mucoepidermoid, and adenoid cystic carcinoma) grow locally extremely slowly and may be mistaken for benign tumors. ${ }^{14}$ Zbaren et al found clinical signs of malignancy, such as pain, facial palsy, and enlarged lymph nodes, in only approximately $30 \%$ of the patients with parotid gland malignancies. ${ }^{15,16}$ Van der Poorten et al stated that facial nerve weakness or paralysis is present in up to $25 \%$ of patients, independently of $\mathrm{T}$ classification, whereas localized pain in the parotid region tends to appear more often (in up to $44 \%$ ). ${ }^{17}$ These literature reports point to the fact that, at least at the initial stage, a by no means negligible number of cases with malignant tumors do not show any clinical evidence of malignancy, even in the eyes of an experienced clinician.

Conversely, painful parotid lesions - in rare cases with facial nerve paresis, suspicious imaging characteristics (blurred tumor margins on ultrasound or magnetic resonance imaging), and even squamous metaplasia on diagnostic cytology - frequently prove to be infected benign tumors of the parotid gland (most commonly adenolymphomas). ${ }^{18,19}$
Consequently, there is a clear potential for a false working hypothesis in preoperative diagnostics in such cases. The aforementioned literature data and our clinical experience show that clinical and imaging approaches can often prove to be demanding in cases of a parotid gland tumor.

A study of the relevant literature shows that unexpected malignancy on definitive histology after minimal invasive surgical modalities on the parotid gland appears not to be particularly rare. McGurk et al demonstrated that 32 of 662 clinically "benign" cases (4.8\%) were subsequently found to have malignant histologies, ${ }^{9}$ with two-thirds of them being low-grade carcinomas. Furthermore, 12 of these 32 cases were primarily operated on by means of extracapsular dissection, ${ }^{9}$ although without oncologic compromises in comparison with the cases receiving the more radical surgical modality (superficial parotidectomy). In another study, Nnochiri et al found a $4 \%$ rate of unexpected malignancies out of 539 clinically unsuspicious cases. ${ }^{20}$ In our study sample, primary malignancies of the parotid gland were detected in 30 of $878(3.4 \%)$ cases, with low-grade malignancies in 22 of $30(73.3 \%)$ cases.

In cases without preoperative suspicion of malignancy and favorable tumor entities (single and mobile lesion with superficial location in the parotid gland), we have increasingly chosen to perform a primary extracapsular dissection of the lesion in our department in recent years. All cases with unsuspected primary parotid malignancy after extracapsular dissection on definitive histology were discussed in our interdisciplinary tumor board. In 25 of our 30 study cases, a completion parotidectomy (resection of the remaining parotid gland tissue) and an ipsilateral elective neck dissection were performed after a maximum interval of 10 days. The need for adjuvant radiotherapy was also discussed in our interdisciplinary tumor board. Of these 25 cases, 5 cases were managed by means of adjuvant radiotherapy (because of microscopic venous invasion [V1] status, positive nodal disease $[\mathrm{pN}+]$, diagnosis of an adenoid cystic carcinoma, a high-grade adenocarcinoma, and a carcinosarcoma), whereas 1 further case received radiochemotherapy after recurrence of a primary "low-grade" acinic cell carcinoma with a secondary high-grade transformation. In 5 cases, a "wait and see" policy was adopted at the patients" request after primary extracapsular dissection with tumorfree margins on definitive histology.

Over the last 12 years of experience in minimal invasive surgery of the parotid gland, one important question has progressively emerged as a part of internal audit: Is it actually that bad for the patient if a malignant tumor of the parotid gland is primarily resected, due to preoperative lack of suspicion of malignancy, by means of extracapsular dissection? Do we cause irreversible damage to such patients if we begin tumor therapy with the most conservative surgery possible?

First of all, as mentioned before, a significant number of cases $(n=22)$ in the initial group of all malignant tumors being primarily managed with extracapsular dissection had tumors without primary parotid origin (eg, lymphomas or 
melanomas). In those cases, extracapsular dissection served as a diagnostic tool and did not prohibit further treatment of the patient (determined in our interdisciplinary tumor board) in our department or by other medical specialties.

Second, the majority of our cases (22 of 30) were lowgrade tumors, which is consistent with results of McGurk et $\mathrm{al},{ }^{9}$ so that one might assume that primarily low-grade cases more frequently masquerade as benign lumps. Interestingly, further tumor cells were detected in the histology specimens of the parotid in only 3 of the 25 cases treated with revision surgery. Only 1 of these 3 cases had a low-grade tumor. Remarkably, only 1 of the 25 cases that received an ipsilateral neck dissection had neck metastases on definitive histology (pN1 status), suggesting an occult metastasis rate of $4 \%$. One could argue that a vigilant "wait and see" policy, in combination with high patient compliance, might have revealed the regional metastasis in this case, perhaps with no negative impact on the patient's survival. Considering the lack of prospective randomized studies showing a clear benefit from more radical procedures - provided that the tumor has been adequately resected on primary surgery (with healthy, tumor-free margins on the specimen on definitive histology) ${ }^{17}$ - one might possibly claim that an extracapsular dissection might be eventually oncologically sufficient as a sole surgical therapy for carefully selected low-grade, nonaggressive, adequately removed cases by patients with high compliance. Interestingly, our analysis showed excellent oncologic results throughout (5-year disease-free survival, 100\%; local control, 96.7\%), which is in accordance with the relevant literature. ${ }^{21}$ Perhaps this might lie in the behavior of these, most often low-grade tumors and not in the surgical modality chosen. Given a mean follow-up of 55.3 months (16-101 months) and the fact that, according to the relevant literature, more than two-thirds of recurrences occur in the first 3 years, ${ }^{22}$ we believe that this preliminary result may not be that discouraging. However, given the prolonged latency and the notoriously indolent behavior of many types of parotid gland malignancies, we undoubtedly need a longer follow-up to reach a safer conclusion in such cases. $^{21}$

From a functional point of view, our study shows that an extracapsular dissection detecting an unsuspected malignancy is not associated with a higher risk of a poor outcome: 28 of 30 cases had normal facial nerve function after surgery (House-Brackmann I); the other 2 cases had a slight permanent paralysis (House-Brackmann II). Four of 25 patients had a temporary slight facial palsy (HouseBrackmann II) postoperatively. In cases in which revision surgery was performed, this was done within a maximum of 10 days, before severe scarring processes had begun. Expectedly, extracapsular dissection offered ideal conditions for the facial nerve during completion surgery, because the nerve was not exposed during the primary operation and thus not bound up in scar tissue. ${ }^{23}$

As mentioned before, our research revealed 30 of 878 (3.4\%) cases with a preoperatively "false" working hypothesis.
Of 878 patients, 25 (2.8\%) had to undergo an additional surgical therapy, according to our protocol, which might not have been necessary if suspicion of malignancy had been raised at a preor intraoperative stage and further diagnostics (FNAC, CNB, or frozen section) had been performed. These patients represent the "penalty" paid for saving 853 patients (in the same period) the need to undergo a more radical and risky procedure for "true" benign tumors.

Undoubtedly, this emotional, psychosocial, and often financial distress to patients caused by a second operation so soon afterward is by no means negligible and lies at the center of our attention. One could claim that preoperative FNAC/CNB or frozen section in every case of parotid gland tumors could enhance diagnostic accuracy and reduce the need for further surgery. This topic is being intensely discussed in the relevant literature and is still a matter of controversy. Salivary gland tumors are relatively uncommon entities that are not regularly encountered by most cytopathologists, only a few of whom ever acquire the experience necessary to diagnose such tumors with confidence and high accuracy. The 24 histologic types, ${ }^{17}$ the cytologic overlap among many salivary gland malignant neoplasms, the low-grade nuclear morphologic features on cytology in many cases, and the dependence of the quality of FNAC on the retention of tissue architectural features all account for the inherent limitations of this diagnostic method. FNAC, with a mean sensitivity of $75 \%,{ }^{24,25}$ has a relatively high risk of false results for malignant tumors. CNB preserves the histologic architecture of the tissue sample, allows some diagnoses that are not possible on the basis of cytology alone (eg, capsular invasion) as well as immunohistochemical analysis, and in this way can offer an advantage over FNAC. Diagnostic accuracy of the aforementioned diagnostic examinations could be improved through the additional use of frozen sections, which, however, requires the availability of an experienced, competent pathologist who has a detailed knowledge of these difficult, often complex pathologic conditions.

As mentioned before, 25 of 878 (2.8\%) patients had to undergo additional surgical therapy, according to the department's previous protocol and strategy to date. If we selfcritically concede that secondary surgery might not have been as necessary or perhaps might constitute overtreatment in the study cases where no further tumor cells in the parotid specimen or no cervical metastases on the neck specimen were found, then secondary surgery would have been indicated in only 4 of 878 cases $(0.45 \%)$. Consequently, we have come to the conclusion that patient distress might be better reduced by being more conservative in our thinking regarding the indications for secondary surgery (the latter, however, only in combination with close patient follow-up). In our view, the extreme rarity of this circumstance seems to justify critical thinking toward less radical surgical treatments. According to these preliminary results and with critical consideration of our short follow-up, the majority of the study cases could be candidates for a "wait and see" approach. 
Furthermore, it is interesting to mention that these "false negative" cases were distributed homogeneously over the years, without any tendency to decrease toward the last years of our study. This might be explained by the influence of 2 opposing factors: On one hand, a change in philosophy with a shift toward less radical surgery over the years has led to the increasing performance of extracapsular dissections in our department; on the other, an increasing "suspicion index" and increased experience in ultrasound examination could contribute to a decrease in the number of such cases. The need to find a balance between these opposing forces may mean that it will not be easy to completely eliminate these cases in the future.

In conclusion, our study concentrated on cases in which there was no preoperative clinical, imaging, or intraoperative suspicion of malignancy. According to the relevant literature and our experience, detecting malignancy on definite pathology is a rarity in these cases. Beginning with the "false" working hypothesis and performing an extracapsular dissection in unsuspected cases seems to have no adverse impact on patients' survival or postoperative quality of life in cases in which definitive histology reveals malignancy. Undoubtedly, the small number of cases and the short follow-up constitute limitations of our study, and the derived data should be verified through a larger population study with a longer follow-up.

\section{Acknowledgments}

The authors wish to thank Ms Petra Güthlein and Mr Philipp Grundtner for their assistance in the collection and analysis of the data.

\section{Author Contributions}

Konstantinos Mantsopoulos, contribution to conception and design of the work, acquisition, analysis and interpretation of data for the work; drafting the work and revising it critically for important intellectual content; final approval of the version to be published; agreement to be accountable for all aspects of the work; Stylianos Velegrakis, contribution to conception and design of the work, acquisition, analysis and interpretation of data for the work; drafting the work and revising it critically for important intellectual content; final approval of the version to be published; agreement to be accountable for all aspects of the work; Heinrich Iro, contribution to conception and design of the work, acquisition, analysis and interpretation of data for the work; drafting the work and revising it critically for important intellectual content; final approval of the version to be published; agreement to be accountable for all aspects of the work.

\section{Disclosures}

Competing interests: None.

Sponsorships: None.

Funding source: None.

\section{Supplemental Material}

Additional supporting information may be found at http://otojournal .org/supplemental.

\section{References}

1. Parkin DM, Bray F, Ferlay J, Pisani P. Global cancer statistics, 2002. CA Cancer J Clin. 2005;55:74-108.

2. Eveson JW, Auclair PL, Gnepp DR. Tumors of the salivary glands: introduction. Head and neck tumours. In: Barnes EL, Eveson JW, Reichart P, Sidransky D, eds. World Health Organization Classification of Tumours: Pathology and Genetics. Lyon, France: IARC Press; 2005:221-222.

3. Spiro RH. Salivary neoplasms: overview of a 35-year experience with 2,807 patients. Head Neck Surg. 1986;8:177-184.

4. Lim YC, Lee SY, Kim K, et al. Conservative parotidectomy for the treatment of parotid cancers. Oral Oncol . 2005;41: 1021-1027.

5. Rahbar R, Grimmer JF, Vargas SO, et al. Mucoepidermoid carcinoma of the parotid gland in children: a 10-year experience. Arch Otolaryngol Head Neck Surg. 2006;132:375-380.

6. Ferlito A, Shaha AR, Rinaldo A, Mondin V.Management of clinically negative cervical lymph nodes in patients with malignant neoplasms of the parotid gland. ORL $J$ Otorhinolaryngol Relat Spec. 2001;63:123-126.

7. Medina JE. Neck dissection in the treatment of cancer of major salivary glands. Otolaryngol Clin North Am. 1998;31: 815-822.

8. Stennert E, Kisner D, Jungehuelsing M, et al. High incidence of lymph node metastasis in major salivary gland cancer. Arch Otolaryngol Head Neck Surg. 2003;129:720-723.

9. McGurk M, Thomas BL, Renehan AG. Extracapsular dissection for clinically benign parotid lumps: reduced morbidity without oncological compromise. Br J Cancer. 2003;89:16101613.

10. House JW, Brackmann DE. Facial nerve grading system. Otolaryngol Head Neck Surg. 1985;93:146-147.

11. Iro H, Zenk J, Koch M, Klintworth N. Follow-up of parotid pleomorphic adenomas treated by extracapsular dissection. Head Neck. 2013;35:788-793.

12. Boahene DK, Olsen KD, Lewis JE, Pinheiro AD, Pankratz VS, Bagniewski SM. Mucoepidermoid carcinoma of the parotid gland: the Mayo clinic experience. Arch Otolaryngol Head Neck Surg. 2004;130:849-856.

13. Klintworth N, Mantsopoulos K, Zenk J, Psychogios G, Iro H, Bozzato A. Sonoelastography of parotid gland tumours: initial experience and identification of characteristic patterns. Eur Radiol. 2012;22:947-956.

14. Digonnet A, Hamoir M, Andry G, et al. Follow-up strategies in head and neck cancer other than upper aerodigestive tract squamous cell carcinoma. Eur Arch Otorhinolaryngol. 2013; 270:1981-1989.

15. Zbaren P, Zbaren S, Caversaccio MD, Stauffer E. Carcinoma ex pleomorphic adenoma: diagnostic difficulty and outcome. Otolaryngol Head Neck Surg. 2008;138:601-605.

16. Zbaren P, Schupbach J, Nuyens M, Stauffer E, Greiner R, Hausler R. Carcinoma of the parotid gland. Am J Surg. 2003; 186:57-62.

17. Van der Poorten V, Bradley PJ, Takes RP, Rinaldo A, Woolgar JA, Ferlito A. Diagnosis and management of parotid 
carcinoma with a special focus on recent advances in molecular biology. Head Neck. 2012;34:429-440.

18. Mantsopoulos K, Psychogios G, Agaimy A, et al. Inflamed benign tumors of the parotid gland: diagnostic pitfalls from a potentially misleading entity. Head Neck. 2013;37:1-7.

19. Chilla R, Droese M. [Infected cystadenolymphoma. A possible reason for clinical and cytologic diagnostic error "carcinoma"']. HNO. 1993;41:446-448.

20. Nnochiri CC, Watkin GT, Hobsley M. Unremarkable parotid tumours that prove to be malignant. Br J Surg. 1990;77:917-918.

21. Terhaard $\mathrm{CH}$, Lubsen $\mathrm{H}$, Van der Tweel I, et al. Salivary gland carcinoma: independent prognostic factors for locoregional control, distant metastases, and overall survival: results of the Dutch head and neck oncology cooperative group. Head Neck. 2004;26:681-692.
22. Bell RB, Dierks EJ, Homer L, Potter BE. Management and outcome of patients with malignant salivary gland tumors. $J$ Oral Maxillofac Surg. 2005;63:917-928.

23. McGurk M, Combes J. Controversies in the Management of Salivary Gland Disease. Oxford, England: Oxford University Press; 2012.

24. Fakhry N, Santini L, Lagier A, Dessi P, Giovanni A. Fine needle aspiration cytology and frozen section in the diagnosis of malignant parotid tumours. Int J Oral Maxillofac Surg. 2014;43:802-805.

25. Zbaren P, Guelat D, Loosli H, Stauffer E. Parotid tumors: fine-needle aspiration and/or frozen section. Otolaryngol Head Neck Surg. 2008;139:811-815. 